\title{
The Mirror Reversal Mechanism on Black Hole Collapse and Singularity Eruption in Cosmic Continuum
}

Xijia Wang ( $\sim$ xijiawang@hunnu.edu.cn )

Hunan Normal University https://orcid.org/0000-0002-0302-7589

\section{Physical Sciences - Article}

Keywords: Cosmic continuum, Planck sphere, Black hole collapse, Singularity eruption, Mirror reversal

Posted Date: November 1st, 2021

DOI: https://doi.org/10.21203/rs.3.rs-958444/v3

License: (c) (i) This work is licensed under a Creative Commons Attribution 4.0 International License.

Read Full License 


\title{
The Mirror Reversal Mechanism on Black Hole Collapse and Singularity Eruption in Cosmic Continuum
}

\author{
Xijia Wang \\ College of Mathematics and Statistics, Hunan Normal University, Changsha, China, 410081 \\ Email: xijiawang@hunnu.edu.cn \\ ORCID: 0000-0002-0302-7589
}

\begin{abstract}
In the Big Bang Theory and the Black Hole Theory, the existing laws of physics all fail at the singularity, and the singularity has become a blind spot in the existing scientific theories. In Cosmic continuum, the cosmic system collapse into a Schwarzschild black hole under the action of a strong gravitational field, and the Planck spheres at the center of the black hole continues to collapse into dark mass bodies, forming dark celestial body and singularity. The Schwarzschild radius is the upper limit of a black hole, and the Planck sphere is the lower limit of a black hole. The singularity is the conversion point between the old and new cosmic systems. The singularity erupts the Planck spheres under the action of a strong gravitational field, and the Planck spheres expands outward to form a new cosmic system. The Planck sphere is both the end of the old cosmic system and the starting point of the new cosmic system. The black hole collapse and the singularity eruption are mirror images of each other. The Planck sphere is the front of the mirror, and the singularity is the back of the mirror.
\end{abstract}

Keywords: Cosmic continuum, Planck sphere, Black hole collapse, Singularity eruption, Mirror reversal

\section{Introduction}

The Big Bang theory believes that the observable universe was created by a big bang that occurred at a dense and hot singularity about 13.8 billion years ago. With the continuous expansion after the Big Bang, the universe evolves in temperature from hot to cold and matter from dense to sparse[1].

In modern cosmology, black holes are formed by the collapse of celestial bodies or cosmic systems. The Schwarzschild radius $R_{s}=\frac{2 G M}{c^{2}}$ is the upper limit of the black hole. Where $G$ is the gravitational constant, $c$ is the speed of light, and $M$ is the mass of the celestial body. At the center of the black hole, it will collapse to form a singularity of infinite mass, density, temperature, and space-time curvature, and infinitely small volume.

Planck unit theory believes that the mass of the ground state particles cannot be greater than the Plank mass $m_{p}$; the energy cannot be greater than or equal to the Planck energy $E_{p}$, otherwise it will collapse into a black hole; Planck time $t_{p}$ is an observable event Minimum process time; Planck length $l_{p}$ is a measure of Plank's quality black hole, unable to distinguish events within a distance less than Planck length; unable to describe events occurring within Planck time when the universe was born, etc. [2]-[10]. 
In the Planck unit system, Planck mass $m_{p}=\sqrt{\frac{h c}{G}}$, Planck energy $E_{p}=\sqrt{\frac{h c^{5}}{G}}$, Planck time $t_{p}=\sqrt{\frac{h G}{c^{5}}}$, Planck length $l_{p}=\sqrt{\frac{h G}{c^{3}}}$. Where $h$ is Planck constant.

However, both the Big Bang theory and the black hole theory face the same paradox: a singularity is a point that exists and does not exist, and all known laws of physics fail at the singularity.

\section{Derivation}

Cosmic continuum is a mathematical continuum theoretical model of the universe [11]-[22]. This article uses the basic concepts and axiom systems in [17]. The following lemmas are all quoted from [18].

Cosmic Continuum Theory believes that the universe is a continuum consisting of an existence continuum and an existing dimension continuum. The existence continuum is composed of mass bodies, energy bodies and dark mass bodies. The existing dimension continuum is composed of space, time and dark space. Space, time, and dark space are the existence dimensions of mass bodies, energy bodies, and dark mass bodies, respectively. The mass body, the energy body, and the dark mass body are respectively composed of particles, quantum and dark particles.

In Cosmic continuum, in addition to known celestial bodies such as planets, stars, and black holes, there is also a dark celestial body in the universe. Its existence quantity is dark mass and its existence dimension is dark space.

Concept 1: Planck sphere refers to a space spheres whose diameter is the Planck length $l_{p}$.

Concept 2: Black hole refers to a celestial body with the Schwarzschild radius $R_{s}$ as the upper limit and the Planck sphere as the lower limit.

Concept 3: Dark celestial bodies refer to celestial bodies composed of dark mass bodies.

Lemma 1: When the mass of particles reaches Planck mass $m_{p}$, the particles are converted into quantum and the mass is converted into energy (proof see [18]).

Lemma 2: When quantum energy reaches Planck energy $E_{p}$, quantum is transformed into dark particles and energy is converted into dark mass (proof see [18]).

Lemma 3: Planck mass $m_{p}$ is equivalent to Planck energy $E_{p}: m_{p} \equiv E_{p} \quad$ (proof see [18]).

Lemma 4: Planck time $t_{p}$ is equivalent to Planck length $l_{p}: t_{p} \equiv l_{p} \quad$ (proof see [18]).

Corollary 1: When the amount of Planck sphere reaches $e \equiv m_{p} \equiv E_{p}$, the Planck sphere collapses into dark particles.

Proof: If the existence quantity of Planck sphere reaches $e \equiv m_{p}$, according to Lemma 1, when the mass of the particle reaches Planck mass, that is, $e \equiv m_{p}$, the particle is transformed into quantum, 
and mass $m_{p}$ will be transformed into energy. According to Lemma 3, the Planck mass $m_{p}$ is equivalent to the Planck energy $E_{p}: m_{p} \equiv E_{p}$. According to Lemma 2, when the energy of a quantum reaches Planck energy $E_{p}$, the quantum is transformed into dark particles, and the energy is transformed into dark mass. If the existence quantity of Planck sphere reaches $e \equiv E_{p}$, according to Lemma 2, when the energy of the quantum reaches the Planck energy $E_{p}$, the quantum is transformed into dark particles, and the energy is transformed into dark mass. In summary, when the existence quantity of Planck sphere reaches $e \equiv m_{p} \equiv E_{p}$, the Planck sphere collapse into dark particles. Q.E.D.

Corollary 1 shows that the Planck sphere is the lower limit of a black hole, and the Planck mass and Planck energy are the limit mass and limit energy of the Planck sphere, respectively.

Concept 4: Planck particles are Planck spheres that collapse into dark particles.

According to Corollary 1 , the existence of Planck particles is the dark mass $D, D \equiv m_{p} \equiv E_{p}$.

Concept 5: Planck dark mass $D_{p}$ refers to the dark mass of Planck particles.

According to Concept 4, Planck dark quality $D_{p}=D \equiv m_{p} \equiv E_{p}$.

Concept 6: Planck existence quantity $e_{p}$ refers to the existence quantity of Planck particles.

According to Corollary 1 and Concept 4, Concept 5, Planck existence quantity $e_{p} \equiv m_{p} \equiv E_{p} \equiv D_{p}$

Corresponding to the Planck unit of the existing quantity, the Planck dimension metric $W_{p}$ can also be defined as the dimension metric of the Planck particle, the Planck dark space metric $g_{p}$ is the dark space degree of the Planck particle, and $W_{P} \equiv t_{P} \equiv l_{p} \equiv g_{p}$.

Corollary 2: The limit density of Planck sphere is $\rho_{\max }=\frac{e_{p}}{V_{p}}$, where $V_{p}=\frac{4}{3} \pi\left(\frac{l_{p}}{2}\right)^{3}$.

Proof: According to Corollary 1 , the limit mass-energy of the Planck sphere is $e_{\max } \equiv m_{p} \equiv E_{p}$. According to Concept $6, e_{p} \equiv e_{\max }$. According to Concept 1, the Planck sphere refers to a sphere with a diameter of Planck length $l_{p}=\sqrt{\frac{h G}{c^{3}}}$, so the volume of the Planck sphere is 
$V_{p}=\frac{4}{3} \pi\left(\frac{l_{p}}{2}\right)^{3}$. Let $e$ be the existence quantity of Planck spheres, then the limit density of Planck spheres $\rho_{\max }=\lim _{e \rightarrow e_{\max }} \frac{e}{V_{p}}=\frac{e_{\max }}{V_{p}}=\frac{e_{p}}{V_{p}}$, where $V_{p}=\frac{4}{3} \pi\left(\frac{l_{p}}{2}\right)^{3}$. Q.E.D.

Corollary 3: In the process of a black hole collapse, every Planck time $t_{p}$ collapses a Planck sphere, the existence quantity collapse speed is $P_{-e}=-\frac{e_{p}}{t_{p}}$, and the negative sign " $-"$ indicates the collapse.

Proof: According to Concept 1, Planck sphere refers to a space spheres whose diameter is the Planck length $l_{p}$. According to Lemma 4, Planck time $t_{p}$ is equivalent to Planck length $l_{p}$ : $t_{p} \equiv l_{p}$. Therefore, the collapse of a Planck sphere is equivalent to the collapse of a Planck time. According to Corollary 1 , when the Planck sphere reaches limit existence quantity $e_{\max } \equiv m_{p} \equiv E_{p}$, the Planck sphere collapses into a dark particle. According to Concept 4, Planck particle is a Planck sphere that collapses into a dark particle, and its existence quantity is $D \equiv e_{\max }$. According to Concept 5, $D_{p} \equiv D \equiv e_{\max }$. According to Concept 6, Planck existence quantity $e_{p} \equiv D_{p}$. Once the Planck sphere at the center of the black hole begins to collapse toward the dark mass, it will continue to collapse until it no longer collapses. Therefore, during the collapse of the black hole, every Planck time $t_{p}$ collapses a Planck sphere, and the velocity of the existence quantity collapse is $P_{-e}=-\frac{D}{t_{p}}=-\frac{e_{\max }}{t_{p}}=-\frac{e_{p}}{t_{p}}$, and the negative sign " - " means collapse. Q.E.D.

Corollary 4: In the process of a black hole collapse, the space collapses in a spherical shape with the Planck sphere as the center, and the collapse radius is $R=-n l_{p}$, and the negative sign " - " means collapse.

Proof: Because the Planck sphere is spherical, when the Planck sphere collapses, the space also collapses in a spherical shape with the Planck sphere as the center. That is, in a Planck time $t_{p}$, the collapsed space around the sphere is the Planck length $l_{p}$, so the space collapse radius is $r=l_{p}$. According to Corollary 3, during the collapse of the black hole, every Planck time $t_{p}$ collapses a Planck sphere, so the space collapse radius is $R=-n r=-n l_{p}$, where $n=\frac{t}{t_{p}}$ represents the 
$n$th Planck time, $t$ represents the collapse time, and the negative sign " - " means collapse. Q.E.D.

Concept 7: Singularity refers to a dark celestial body formed by the collapse of a black hole.

Concept 7 shows that the singularity is a special kind of dark celestial body.

Corollary 5: In the process of a singularity eruption, every Planck time $t_{p}$ erupts a Planck sphere, and the eruption velocity of the existence quantity is $P_{e}=\frac{e_{p}}{t_{p}}$.

Proof: Same as Corollary 3, just replace the black hole collapse process with the singularity eruption process. Q.E.D.

Corollary 6: In the process of a singularity eruption, the space expands in a spherical shape with the Planck sphere as the center, and the expansion radius is $R=n l_{p}$, where $n=\frac{t}{t_{p}}$ represents the $n$th Planck time and $t$ represents the expansion time.

Proof: Same as Corollary 4, just replace collapse with expansion. Q.E.D.

\section{Result}

Mirror reversal theorem: The black hole collapse and the singularity eruption are mirror reversals of each other.

Proof: According to Corollary 3 and Corollary 4: In the process of a black hole collapse, every Planck time $t_{p}$ collapses a Planck sphere, the existence quantity collapse speed is $P_{-e}=-\frac{e_{p}}{t_{p}}$. The space collapses in a spherical shape with the Planck sphere as the center, and the collapse radius is $R=-n l_{p}$, and the negative sign " $-"$ means collapse. According to Corollary 5 and Corollary $6: \ln$ the process of a singularity eruption, every Planck time $t_{p}$ erupts a Planck sphere, and the eruption velocity of the existence quantity is $P_{e}=\frac{e_{p}}{t_{p}}$. The space expands in a spherical shape with the Planck sphere as the center, and the expansion radius is $R=n l_{p}$, where $n=\frac{t}{t_{p}}$ represents the $n$th Planck time and $t$ represents the expansion time. The black hole collapse and the singularity eruption are like the same process inside and outside the mirror, so the black hole collapse and the singularity eruption are mirror images of each other. Q.E.D.

Mirror reversal theorem shows that the mechanism of the collapse and the expansion of the universe system is: the old universe system collapses into a black hole, and the Planck sphere at the center of the black hole continues to collapse to form a singularity; the singularity erupts the Planck sphere, and the Planck sphere expands to generate a new cosmic system. The Planck sphere is both the end of the old cosmic system and the starting point of the new cosmic system.

It should be pointed out that the mirror inversion theorem is only a mechanism for the collapse and 
expansion of the universe system, and any universe system is only a component of the entire universe.

\section{Discussion}

In December 2011, more than 80 high school students in the United States and their teachers simply multiplied the Planck length by 2 again and again, to 202 times to reach the size of the observable universe.

Bruce Camber immediately devoted himself to promoting the exploration of this "big board-small universe" project, and made a chart of the universe using the geometric progression method with a common ratio of 2, in order to present the expansion process from the Planck scale to the observable universe.

Following the research of the project, Bruce Camber proposed a bolder vision: the universe starts from a singularity, and quiet expansion on "Continuity•Symmetry•Harmony" under the action of thrust for perfection. The thrust for perfection comes from one Planck sphere per Planck time. Bruce Camber's perfect thrust coincides with the singularity eruption mechanism of the Cosmic continuum.

According to the chart of the universe of the "big board-small universe" project, the first 3 data of step 202 are as follows [23]:

$$
\begin{aligned}
& t_{202}=t_{p} \cdot 2^{202} \approx 5.3911613 \times 10^{-44} \times 2^{202} \approx 3.4654 \times 10^{17} \quad(\mathrm{~s}) \\
& l_{202}=l_{p} \cdot 2^{202} \approx 1.61622938 \times 10^{-35} \times 2^{202} \approx 1.038 \times 10^{26} \quad(\mathrm{~m}) \\
& m_{202}=m_{p} \cdot 2^{202} \approx 2.17647051 \times 10^{-8} \times 2^{202} \approx 1.399 \times 10^{52} \quad(\mathrm{~kg})
\end{aligned}
$$

The first data represents the age of the observable universe up to step 202, which can be converted to $t_{202} \approx 10.98$ billion years.

The second data represents the size of the observable universe up to step 202, which can be converted to $l_{202} \approx 1.098 \times 10^{10}$ light-years, I.e. 10.98 billion light-year. According to Corollary 6 , the formula for calculating the radius of the observable universe is $R=n l_{p}$, so $l_{202}$ should be the radius of the observable universe. Therefore, in step 202, the observable universe has a radius of $l_{202} \approx 1.098 \times 10^{10}$ light-years and a diameter of $2 \times l_{202} \approx 2.196 \times 10^{10}$ light-years.

The third data represents the mass of the observable universe up to step 202. According to Corollary 5, during the eruption of the singularity, the eruption velocity of the existence quantity is $P_{e}=\frac{e_{p}}{t_{p}}$, so in step 202, the existence quantity of the observable universe is $e_{202} \equiv 1.399 \times 10^{52}$ kilograms. It should be reminded that this existence quantity does not include the existence quantity of the singularity in the center of the observable universe that has not yet erupted.

The calculation method of the above three data is consistent with the Cosmic continuity, and is calculated according to a Planck sphere per Planck time.

It is not difficult to find that the observable universe age in step 202 has not reached the age of the universe calculated according to the standard model at about 13.8 billion years, indicating that the expansion of the universe has not stopped at step 202. And to step 203, the above 3 data all need to 
be multiplied by 2 , and they are:

$$
\begin{aligned}
& t_{203}=t_{p} \cdot 2^{203}=2 \times t_{202} \approx 21.96 \text { (billion years) } \\
& l_{203}=l_{p} \cdot 2^{203}=2 \times l_{202} \approx 2.196 \times 10^{10} \text { (light-years) } \\
& m_{203}=m_{p} \cdot 2^{203}=2 \times m_{202} \approx 2.798 \times 10^{52}(\mathrm{~kg})
\end{aligned}
$$

At this time, the age of the observable universe is $t_{203} \approx 21.96$ billion years, the diameter is $2 \times l_{203} \approx 4.392 \times 10^{10}$ light-years, and the existence quantity is about $e_{203} \equiv 2.798 \times 10^{52}$ kilograms.

There are two other data in the chart of the universe, charge and temperature, which are not used here because they are inconsistent with the Cosmic continuum.

In fact, not only the cosmological diagram, but also the existing basic theories of cosmology still have many unresolved problems: First, where does the increasing mass of the observable universe come from? Second, why is there a Planck sphere every Planck time? Third, is the singularity dense and hot or cold and dark? Fourth, was the observable universe created by the Big Bang or was it quietly expanding? Fifth, how do the four interactions occur? Sixth, where are the dark matter and dark energy in the universe?[24]-[42]

Below, let us use the universe continuum and its mirror reversal theory to answer these puzzles one by one.

First, the ever-increasing existence quantity of the observable universe comes from the eruption of singularities. The singularity is formed by the collapse of the old cosmic system. After the old cosmic system collapsed into a black hole, the Planck sphere at the center of the black hole continued to collapse to form a singularity. The existence quantity of singularity is determined by the existence quantity of singularity that the old cosmic system collapses to.

Second, time and space belong to the category of existential dimensions. Since the Planck time is equivalent to the Planck length, and the Planck sphere is a space sphere with the Planck length as the diameter, the black hole collapse and singularity eruption are both a Planck sphere per Planck time , And the existence quantity collapse and eruption speeds, and the space collapse and expansion speeds are uniform.

Third, the singularity is a kind of dark celestial body, and its existential dimension is dark space. Therefore, the singularity does not occupy space, let alone the concepts of density and temperature. The reason why a singularity is considered to be a point that both exists and does not exist, and all known laws of physics fail at the singularity because the singularity is beyond the cognitive scope of the existing theories of physics foundation.

Fourth, the density of the Planck sphere erupted by the singularity is the limit density, which is the maximum density in the observable universe. The temperature of the Planck sphere erupted by the singularity should also be the limit temperature, that is, the Planck temperature. Therefore, the observable universe is a spherical expansion of a dense and hot Planck sphere.

Fifth, after the collapse of the old cosmic system into a singularity, the singularity only has gravitational interaction with the outside world. After the singularity erupts the Planck sphere, during the expansion process, due to the background radiation, quantum fluctuations and other factors in 
the entire universe, the new cosmic system generates Symmetry Breaking to form positive and negative charges and particles of matter, resulting in electromagnetic interaction, strong and weak interactions, and evolve into large-scale cosmic structures.

Sixth, the universe is full of existences in the form of dark mass. Celestial bodies composed of dark masses are dark celestial bodies. Dark objects generally exist in the center of the black hole system. The singularity of the observable universe is also a dark celestial body. Dark energy is the gravitational field energy that fills the universe, and it is the source of all energy in the universe.

\section{Conclusion}

The discussion in this article shows that the collapse of the black hole and the eruption of the singularity are mirror images of each other. The Planck sphere is the front of the mirror, and the singularity is the back of the mirror. Celestial bodies or cosmic systems collapse to form black holes, and the Planck sphere at the center of the black hole continuously collapses into dark masses, forming dark celestial bodies and singularities. The singularity erupts the Planck sphere, and the Planck sphere expands with the singularity as the center to form a new cosmic system.

The singularity is the conversion point between the old and new cosmic systems. The singularity is not a point with infinite mass, infinite density, infinite temperature, infinite curvature of space-time, and infinitely small volume. A singularity is a kind of dark celestial body, its existence quantity is dark mass, and its existence dimension is dark space. Since the singularity does not occupy space, there is no concept of density, temperature, and curvature of space-time. The dark mass of a singularity is the sum of the existence quantity of black hole collapsed.

The universe is a continuum composed of an infinite number of cosmic systems including the observable universe. The mass and energy of the observable universe are not produced from the void, but transformed from the dark mass of the singularity; time and space are not born out of nothing, but are transformed from the dark space of the singularity. As long as the singularity is still erupting, the observable universe will not stop expanding.

The authors have no relevant financial or non-financial interests to disclose.

\section{References:}

[1] Chen Jiansheng, Wang Shouwan, Su Hongjun, Yu Yunqiang, Zou Zhenlong. A brief introduction to the theory of stellar evolution and the thermal big bang cosmology. Scientific Chinese, 5: 17-19 (1998).

[2] Planck, Max. "Über irreversible Strahlungsvorgänge". Sitzungsberichte der Königlich Preußischen Akademie der Wissenschaften zu Berlin. 5: 440-480 (1899).

[3] Luis Gonzalez-Mestres, EPJ Web of Conferences. Volume 71, 2nd International Conference on New Frontiers in Physics. (2014) doi: https://doi.org/10.1051/epjconf/20147100062.

[4]Joachim Fischer, Joachim Ullrich. The new system of units. Nature Physics, 12, 4-7 (2016).

[5]Paul F. Kisak. Planck Units: The Fundamental Scale of Cosmology. Createspace Independent Pub, Colorado Springs.

[6] Staff. Birth of the Universe. University of Oregon. Retrieved September 24, 2016.-discusses "Planck time" and "Planck era" at the very beginning of the Universe (2016).

[7] John D. Barrow, The Constants of Nature; From Alpha to Omega - The Numbers that Encode the Deepest Secrets of the Universe. (Pantheon Books. New York, 2002).

[8] P.A.M. Dirac. A New Basis for Cosmology. Proceedings of the Royal Society A. 165 (921): 199-208 
(1938). Bibcode:1938RSPSA.165..199D. doi:10.1098/rspa.1938.0053

[9] Pavšic, Matej. The Landscape of Theoretical Physics: A Global View. (Dordrecht: Kluwer Academic, 2001). pp. 347-352. ISBN 978-0-7923-7006-2.https://arxiv.org/ abs/gr-qc/0610061

[10] Davies, Paul C.; Davis, TM; Lineweaver, CH. Cosmology: Black Holes Constrain Varying Constants. Nature. 418 (6898):602-3(2002). Bibcode:2002Natur.418..602D. doi: 10.1038/418602a. PMID 12167848

[11] Wang, X.J. Scientific poverty and outlet, Science and Management, 4, 28-30 (1990).

[12] Wang, X.J. and Wu, J.X. Unification. (Haitian Publishing House, Shenzhen, 1992).

[13] Wang, X.J. and Wu, J.X. Unification - Deciphering the mysteries of the universe. Tech wave, 11 , 24-26 (1993). Xinhua Digest, 1, 180-182(1994).

[14] Wang, X.J. The Sublimation of Thinking Experiment. Invention and Innovation, 6, 8-9 (1997).

[15] Wang, X.J. and Wu, J.X. To solve the mystery of scientific unity. (Hunan Science and Technology Press, Changsha, 2001).

[16] Wang, X.J. Unification Theory: Challenging Traditional Scientific Norms. Invention and Innovation, 4, 32-33 (2003).

[17] Wang, X.J. Cosmic Continuum Theory: A New Idea on Hilbert's Sixth Problem. Journal of Modern Physics, 9: 1250-1270(2018) . https://doi.org/10.4236/jmp.2018.96074

[18] Wang, X.J. New Discovery on Planck Units and Physical Dimension in Cosmic Continuum Theory. Journal of Modern Physics, 9: 2391-2401 (2018). https://doi.org/10.4236/jmp.2018.914153

[19] Wang, XJ. New Explanation on Essence of Quantum Phenomena and Interactions and the Gravitational Action in Cosmic Continuum Theory. SSRG International Journal of Applied Physics, 7:3: 88-96 (2020). https://doi .org/10.14445/23500301/IJAP-V7I3P114

[20] Wang, X.J. Hilbert's First Problem and the New Progress of Infinity Theory. PREPRINT (Version 3) available at Research Square(6 Aug 2021). https://doi.org/10.21203/rs.3.rs-306991/v3

[21] Wang, X.J. Modern Interpretation of Classic of Changes System. Social Science, 9, 57-59 (1997).

[22] Wang, X.J. Axiomatization of the Symbols System of Classic of Changes: The Marriage of Oriental Mysticism and Western Scientific Tradition. Foundations of Science, 25:315-325(2020). https://doi.org/ 10.1007/s10699-019-09624-5

[23] Chart of the Universe. Worldviews Are Too Limited (2021). https://81018.com/chart/

[24] Jones, Andrew Zimmerman. "Kelvin's "Clouds" Speech." ThoughtCo, (29 Sep 2017). thoughtco.com/kelvins-clouds-speech-2699230

[25] David Gross. The Future of Physics, International Journal of Modern Physics A, 20 (26) :5897-5909 (2005).

[26] Einstein, Albert. On a Heuristic Viewpoint Concerning the Production and Transformation of Light, Annalen der Physik. 17 (6): 132-148 (1905). Bibcode:1905AnP...322..132E, doi:10.1002/andp.19053220607

[27] Kumar, Manjit. Quantum: Einstein, Bohr, and the Great Debate about the Nature of Reality (Reprint ed.). (W. W. Norton \& Company, 2011). pp. 242, 375-376. ISBN 978-0393339888

[28] Einstein, Albert. Does the Inertia of a Body Depend Upon Its Energy Content?, Annalen der Physik 18 (13): 639-641 (1905). Bibcode:1905AnP...323..639E, doi:10.1002/andp.19053231314

[29] Riazuddin. Non-standard interactions(PDF). NCP 5th Particle Physics Sypnoisis. Islamabad: Riazuddin, Head of High-Energy Theory Group at National Center for Physics (December 29, 2009). 1 (1): 1-25 (2009).

[30] Standard Model of Particles and Interactions. jhu.edu. Johns Hopkins University. Archived 
from the original on March 4, (2016). Retrieved August 18, 2016. .gif

[31] Kenneth S. Krane. Introductory Nuclear Physics. (Wiley \& Sons, 1988). ISBN 0-471-80553-X.

[32] Wiringa, R. B.; Stoks, V. G. J.; Schiavilla, R. Accurate nucleon-nucleon potential with charge-independence breaking. Physical Review C, 51: 38(1995).

arXiv:nucl-th/9408016 . Bibcode:1995PhRvC..51...38W. doi:10.1103/PhysRevC.51.38

[33] Meinard Kuhlmann, Physicists debate whether the world is made of particles or fields - or something else entirely, Scientific American, (24 Jul 2013)

[34] Nathaniel Page Stites, M.A./M.S. Light I: Particle or Wave?, Visionlearning. Vol. PHY-1 (3) (2005). http://www.visionlearning.com/library/module_viewer.php?mid=132

[35] R. Colella, A. W. Overhauser and S. A. Werner, Observation of Gravitationally Induced Quantum Interference, Phys. Rev. Lett . 34, 1472-1474 (1975).

[36] Brezger, Björn; Lucia Hackermüller; Stefan Uttenthaler; Julia Petschinka; Markus Arndt; Anton Zeilinger. Matter-wave interferometer for large molecules. Phys. Rev. Lett. 88(10): 100404 (2002). arXiv:quant-ph/0202158 . Bibcode:2002PhRvL..88j0404B. doi:10.1103/PhysRevLett.88.10 0404. PMID 11909334. Archived from the original on 2016-05-21

[37] Gerlich, Stefan; et al. Quantum interference of large organic molecules. Nature Communications,2:263(2011).

Bibcode:2011NatCo...2E.263G.doi:10.1038/ncomms1263. PMC 3104521. PMID 21468015

[38] Bouwmeester, Dik; Pan, Jian-Wei; Mattle, Klaus; Eibl, Manfred; Weinfurter, Harald \& Zeilinger, Anton. Experimental Quantum Teleportation (PDF). Nature. 390 (6660): 575-579 (1997). Bibcode:1997Natur.390..575B. doi:10.1038/37539

[39] Einstein A, Podolsky B, Rosen N; Podolsky; Rosen. Can Quantum-Mechanical Description of Physical Reality Be Considered Complete? (PDF). Phys. Rev. 47 (10): 777-780 (1935). Bibcode:1935PhRv...47..777E. doi:10.1103/PhysRev.47.777

[40] The BIG Bell Test Collaboration . Challenging local realism with human choices. Nature. 557: 212-216 (9 May 2018). doi:10.1038/s41586-018-0085-3

[41] Lee, K. C.; Sprague, M. R.; Sussman, B. J.; Nunn, J.; Langford, N. K.; Jin, X.- M.; Champion, T.; Michelberger, P.; Reim, K. F.; England, D.; Jaksch, D.; Walmsley, I. A. Entangling macroscopic diamonds at room temperature. Science. 334 (6060): 1253-1256 (2 December 2011). Bibcode:2011Sci...334.1253L. doi:10.1126/science.1211914. PMID 22144620. Lay summary

[42]B. P. Abbott et al. (LIGO Scientific Collaboration and Virgo Collaboration). Observation of Gravitational Waves from a Binary Black Hole Merger. Physical Review Letters. 116:6 (2016) 